Classification

Physics Abstracts

$03.20-03.65-05.40$

\title{
Spectral properties of the Laplacian and random matrix theories
}

\author{
O. Bohigas, M. J. Giannoni and C. Schmit \\ Division de Physique Théorique (*), Institut de Physique Nucléaire, \\ B.P. No 1,91406 Orsay Cedex, France
}

(Reçu le 31 juillet 1984, accepté le 11 septembre 1984)

\begin{abstract}
Résumé. - Nous étudions les propriétés de fluctuation des valeurs propres du Laplacien à deux dimensions avec des conditions aux limites de Dirichlet sur un stade. Elles sont consistantes avec les fluctuations des valeurs propres de matrices aléatoires (GOE). Nous faisons la conjecture que ceci est vrai en général, pourvu que la frontière soit telle que le mouvement d'une particule libre réfléchie élastiquement par la frontière (billard) soit un mouvement très chaotique.

Abstract. - We investigate the fluctuation properties of the eigenvalues of the Laplacian in two dimensions with Dirichlet boundary conditions on a stadium. They are found to be consistent with the fluctuations of eigenvalues of random matrices (GOE). It is conjectured that this is true for any boundary such that the motion of a free particle elastically reflected by the boundary is a strongly chaotic motion.
\end{abstract}

The work described in this Letter is a continuation of our investigation started in reference [1] (hereafter referred as I; see also [2]). In section 1, we summarized the reasons for believing that the level fluctuation properties (departures from uniformity) of spectra, as predicted by random matrix theories, specifically by the Gaussian Orthogonal Ensemble (GOE) [3], are expected to be very general. We presented results showing that the level fluctuations of the eigenvalues $E_{n}$ of the Laplacian

$$
\left(\Delta+E_{n}\right) \psi_{n}=0
$$

in two dimensions, with the Dirichlet boundary condition $\psi_{n}=0$ on Sinai's billiard (see Fig. 1), are consistent with GOE-fluctuations. We remember that Sinai's billiard (free point particle elastically reflected by the walls) shows classically a strongly chaotic motion (Bernoulli system) and that in I it has been conjectured that the agreement between GOE fluctuations and level fluctuations of time-reversal invariant quantum systems whose classical analogues are strongly chaotic is a general property. In contrast, for a system whose classical analogue is integrable, for instance a circular billiard, i.e., solutions of equation (1) with Dirichlet boundary conditions on the circle, the spectral fluctuations have been shown to be asymptotically of Poisson type [4]. It should be noticed that, recently, two cases showing convincingly the transition in the quantum spectral fluctuations from Poisson to GOE type, when the corresponding classical Hamiltonian

$\left(^{*}\right)$ Laboratoire associé au C.N.R.S. 


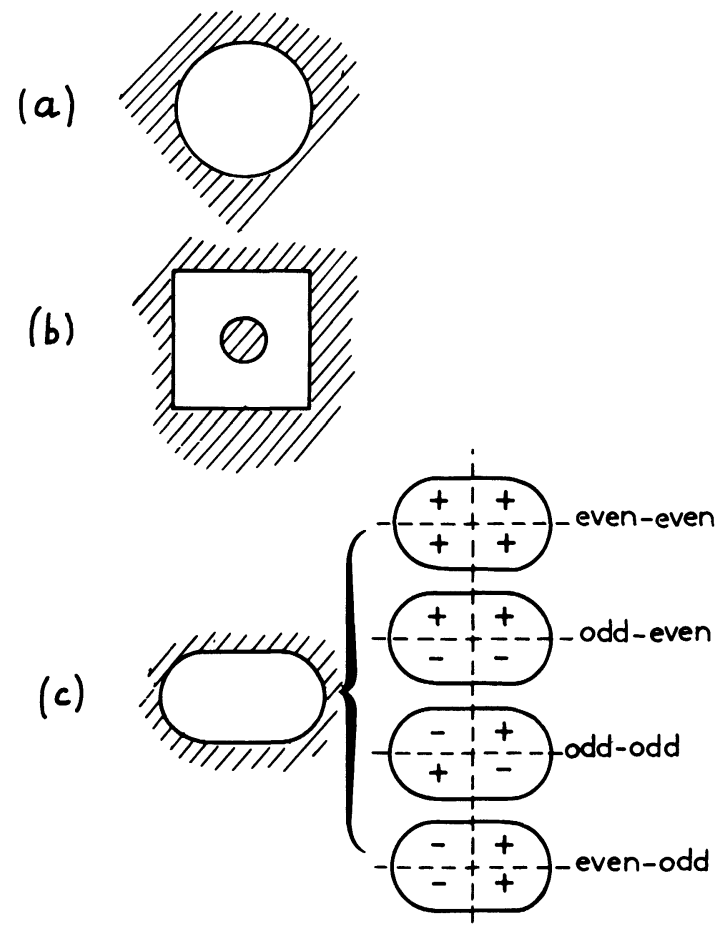

Fig. 1. - Shapes of the boundaries of different membranes whose spectral fluctuations are discussed in the text : a) circle; b) Sinai; c) stadium, with the four symmetry classes of the eigenfunctions.

undergoes a transition from the integrable to the chaotic regime, have been studied [5]. In two dimensions, equation (1) applies not only to the quantum problem of a free particle in a box but also to the classical problem of the transverse vibrations of membranes of different shapes whose boundaries are fixed. In three dimensions it applies, with appropriate changes of the boundary conditions, to the free electromagnetic oscillations in the interior of a cavity.

In the present note we present results for the spectrum of equation (1) in two dimensions with Dirichlet boundary conditions on the stadium : two semi-circles separated by two straight segments (see Fig. 1). It is known that a stadium billiard is classically a Bernoulli system [6] and spectral properties of equation (1) with a stadium boundary have been previously investigated in the pioneering work of McDonald and Kaufman [7], where, among other questions, the problem of the correspondence between ray and wave description was properly emphasized $\left({ }^{1}\right.$ ) (see also Ref. [9]). The results presented here go beyond those reference [7] in several respects : i) we study not only the spacing distribution $p(x)$ between nearest-neighbour levels, which is adequate to study level repulsion (avoidance of clustering of levels) but also other quantities characterizing level fluctuations (spectral rigidity, for instance); ii) the number of computed eigenvalues is significantly larger than in [7], thereby allowing a close comparison with GOE predictions; iii) by computing all the solutions belonging to the four different symmetry classes of the stadium, the role of the discrete symmetries can be investigated. Besides treating a different billiard, this work differs from $I$ in another respect : not only 2-point functions are dealt with, but 3- and 4-point functions are discussed as well.

( ${ }^{1}$ ) After this work has been completed, we received the Ph. D. Thesis of McDonald [8], in which the results of Ref. [7] have been considerably extended. 
We proceed as in I, except that the parameter characterizing the billiard will be kept fixed and that each different symmetry class, labelled by $\alpha$, will be treated separately. $\alpha$ takes four different values corresponding to the even-even, odd-even, odd-odd, even-odd solutions, labelled by $\alpha=++,-+,--,+-$ respectively and one has

$$
\begin{aligned}
& \psi_{++}(x, y)=\psi_{++}(-x, y)=\psi_{++}(x,-y) \\
& \psi_{-+}(x, y)=\psi_{-+}(-x, y)=-\psi_{-+}(x,-y) \\
& \psi_{--}(x, y)=-\psi_{--}(-x, y)=-\psi_{--}(x,-y) \\
& \psi_{+-}(x, y)=-\psi_{+-}(-x, y)=\psi_{+-}(x,-y) .
\end{aligned}
$$

The geometrical symmetry of the problem does not introduce any degeneracy in the spectrum. Once the eigenvalues $E_{i, \alpha}$ have been determined, we unfold the spectrum by the mapping $E_{i, \alpha} \mapsto \varepsilon_{i, \alpha}$ through

$$
\varepsilon_{i, \alpha}=\bar{N}_{\alpha}\left(E_{i, \alpha}\right)
$$

where $\bar{N}_{\alpha}(E)$ represents the smoothed part of the staircase function number of levels $N_{\alpha}(E)$ up to energy $E$ corresponding to the symmetry class $\alpha$. The effect of equation (3) is to map the spectrum $\left\{E_{i}\right\}$ into a spectrum $\left\{\varepsilon_{i}\right\}$ which is on the average uniform and with mean spacing equal to one. To determine $\bar{N}_{\alpha}(E)$ we use the Weyl-type formula [10]

$$
\bar{N}(E)=(1 / 4 \pi)(S E-\mathcal{L} \sqrt{E}+C)
$$

where $S$ is the surface of the boundary, $\mathcal{L}$ its perimeter, and $C$ a constant containing information on the geometrical and topological properties of the domain (curvature and corners of the boundary, connectivity of the surface). Equation (4) applies to the number of eigenvalues up to energy $E$ of (1) with Dirichlet boundary conditions. To determine $\bar{N}_{\alpha}(E)$ we proceed as follows. Let $N_{0}(E)$, $N_{1}(E), N_{-}(E), N_{+}(E)$ denote the number of eigenvalues up to energy $E$ corresponding to the complete stadium, to the right (or left) half of the stadium, to the upper (or lower) half of the stadium and to one quarter of the stadium respectively. One has

$$
\begin{aligned}
N_{0}(E) & =N_{++}(E)+N_{-+}(E)+N_{--}(E)+N_{+-}(E) \\
N_{1}(E) & =N_{+-}(E)+N_{--}(E) \\
N_{-}(E) & =N_{-+}(E)+N_{--}(E) \\
N_{+}(E) & =N_{--}(E)
\end{aligned}
$$

For the functions $N_{0}, N_{1}, N_{-}, N_{+}$, equation (4) is valid. By inverting (5) one has the functions $N_{\alpha}$ in terms of $N_{0}, N_{1}, N_{-}$and $N_{+}$and, applying (4) one obtains $\bar{N}_{\alpha}(E)$. The leading term (surface term) of $\bar{N}_{\alpha}(E)$ is just one fourth of the corresponding term of $\bar{N}_{0}(E)$ and independent of $\alpha$. The $\alpha$-dependence of $\bar{N}_{\alpha}(E)$ comes only through the perimeter and constant terms in equation (4). Although (4) is only asymptotically valid, it can be seen that in practice it applies all over the spectrum, even for small values of $E$.

To determine the eigenvalues we use essentially the numerical technique proposed in reference [11] and applied in [7] : by making an integral dipole representation of the wave function the original differential equation to be solved is transformed into an integral equation. In order to check the numerical accuracy of the results, we have computed the eigenvalues for the circular case, for which the solutions are known to be the square of the zeros of the Bessel functions $J_{v}(X)$ of integer order of the first kind. The eigenvalues are determined with a precision of the order of a few percent of the average spacing. The algorithm may fail in practice when two eigen- 
values are almost degenerate. For instance, for the circular case, around $3 \%$ of the eigenvalues are missed. This fraction is probably less for the case of the stadium. For the stadium we used a ratio of straight line segment length over radius length equal to unity and the first 3200 eigenvalues were computed. A stretch containing the first 1400 eigenvalues of the circle has been computed exactly.

We now turn to the results. The following quantities are discussed :

a) the spacing distribution $p(x)$ between adjacent levels;

b) the average over the spectrum $\bar{\Delta}_{3}(L)$ of $\Delta_{3}(L) . \Delta_{3}(L)$ is defined as follows : take an interval $[\varepsilon, \varepsilon+L] ; \Delta_{3}(L)$ is the least-square deviation of the staircase function $N(\varepsilon)$ giving the cumulative density of the spectrum $\left\{\varepsilon_{i}\right\}$ from the best straight line fitting it;

c) the variance $\Sigma^{2}(L)$, the skewness $\gamma_{1}(L)$ and the excess $\gamma_{2}(L)$ of the number $n(L)$ of points contained in an interval $[\varepsilon, \varepsilon+L] . \Sigma^{2}, \gamma_{1}$ and $\gamma_{2}$ are given in terms of the spectral averages of the square, the cube and the fourth power of $(n(L)-L)$.

For the sake of comparison, corresponding values for Poisson and GOE spectra are also given [12]. $\Sigma^{2}$ and $\bar{\Delta}_{3}$ are given in terms of integrals of the 2-level cluster function whereas $\gamma_{1}$ and $\gamma_{2}$ are given in terms of integrals of the $(2+3)$ - and $(2+3+4)$-level cluster functions. For Poisson spectra, $\Sigma^{2}$ and $\bar{\Delta}_{3}$ increase linearly with $L$, whereas for GOE one has a logarithmic increase (spectrum rigidity).

From the results obtained it can be seen that, in the case of the stadium, the level fluctuations corresponding to each different symmetry class are, to within statistical errors, the same and consistent with GOE predictions. This is illustrated on figure $2 \mathrm{~b}$, where $\bar{\Delta}_{3}(L)$ is given for the four different symmetry classes separately. In order to improve the statistical significance of the results, we may consider, instead of four separate stretches of $\sim 800$ levels, a single stretch obtained by the succession of the four stretches (levels belonging to different symmetries are not mixed).

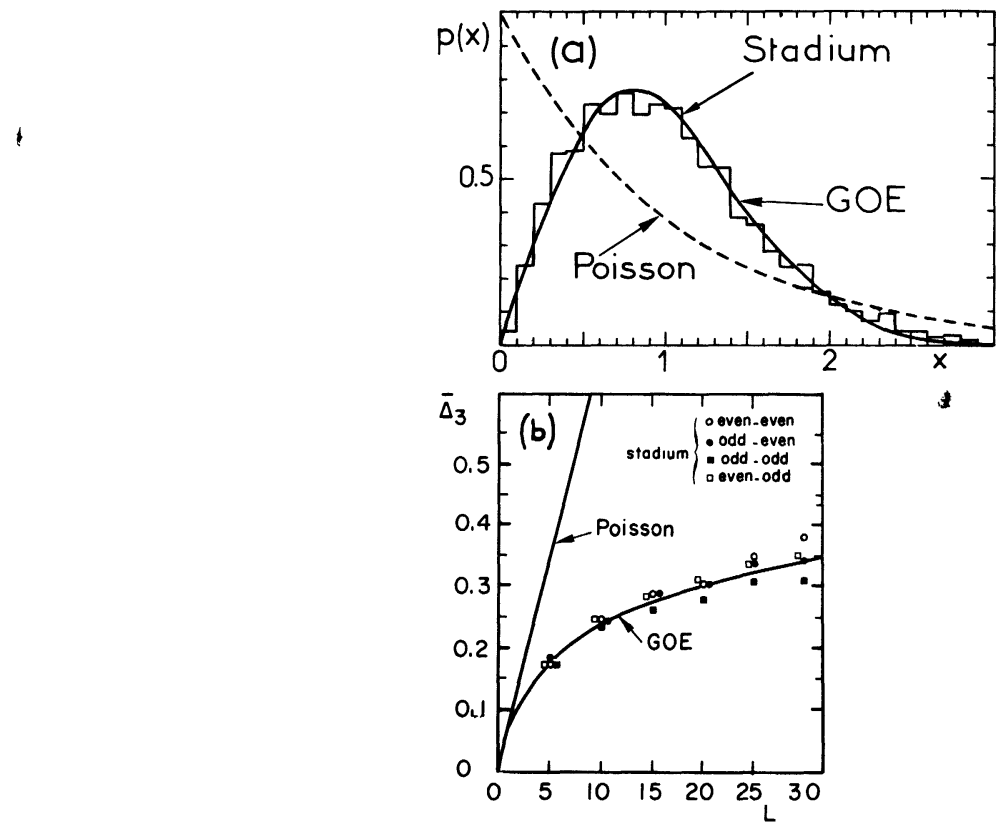

Fig. 2. - Results of level fluctuations for the eigenvalues of the stadium : (a) nearest-neighbour spacing distribution; (b) $\bar{\Delta}_{3}$ as a function of $L$. In (b) results are obtained from the stretch of levels going from the 50th to 800th level for each symmetry class. In (a), to improve the statistics, all spacings corresponding to each symmetry are included. GOE and Poisson predictions are drawn for comparison. 
Results presented in figure $2 \mathrm{a}$ for the spacing distribution and in figure 3 for $\Sigma^{2}, \gamma_{1}$ and $\gamma_{2}$ are obtained in this way and, again, are consistent with GOE predictions. If one considers a stretch of the first 3200 levels, ordered with increasing value of the energy and irrespective of the symmetry class, one obtains the results displayed in figure 4. They agree with the predictions obtained by superposing four uncorrelated GOE spectra each having the same mean spacing. When superposing $n$ uncorrelated sequences of $n$ GOE spectra with the same mean spacing one has, for instance,

$$
\left[\bar{\Delta}_{3}(L)\right]_{\mathrm{mixed}}=\frac{1}{n} \bar{\Delta}_{3}^{\mathrm{GOE}}(L / n)
$$

These results are in perfect analogy with what happens when analysing resonances of the compound nucleus : GOE predictions apply to sequences of levels having the same quantum numbers but when states belonging, for instance, to different parities are not separated, one must consider superpositions of uncorrelated GOE spectra. For comparison, results corresponding to an integrable case, the case of a circular boundary, are also presented. To avoid degeneracies, we consider in fact the semi-circular membrane. Results are shown on figures 5 and 3 and are close to Poissonfluctuations. We interpret the departures from Poisson results as an indication that the asymptotic regime has not yet been attained. That one expects a Poisson spectrum can be understood from the following heuristic argument. One knows that the result of randomly superposing highly

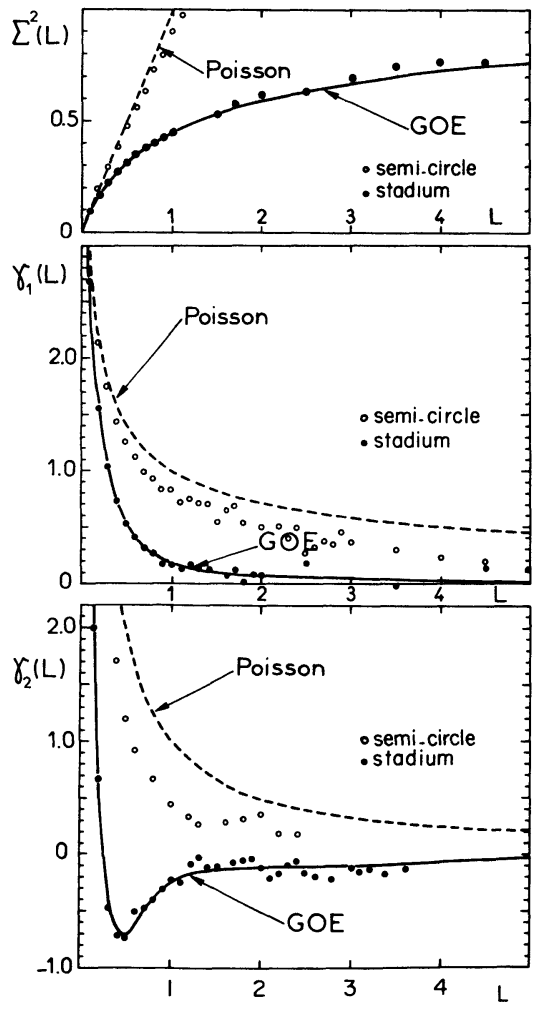

Fig. 3. - Variance $\Sigma^{2}$, skewness $\gamma_{1}$ and excess $\gamma_{2}$ of the number statistic $n(L)$ as a function of $L$, for the semi-circular and stadium membranes. For the semi-circular membrane, the stretch going from the 200th to the 1400 th level has been used. For the stadium, see caption of figure 2a. 


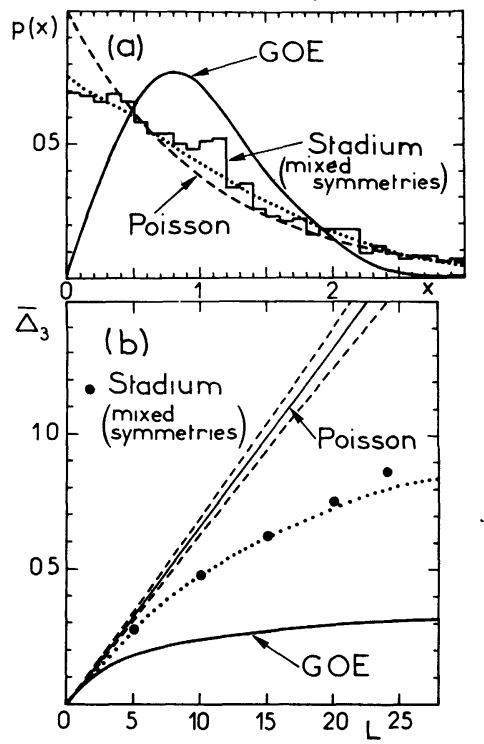

Fig. 4. - Same as in figure 2 using the stretch going from the 200th to the 3200 th eigenvalue, without separating according to the symmetry class. Dashed lines for the $\bar{\Delta}_{3}$-value of Poisson indicate the effect of the finiteness of the sample (one standard deviation). Dot lines indicate results corresponding to the superposition of four uncorrelated GOE spectra.

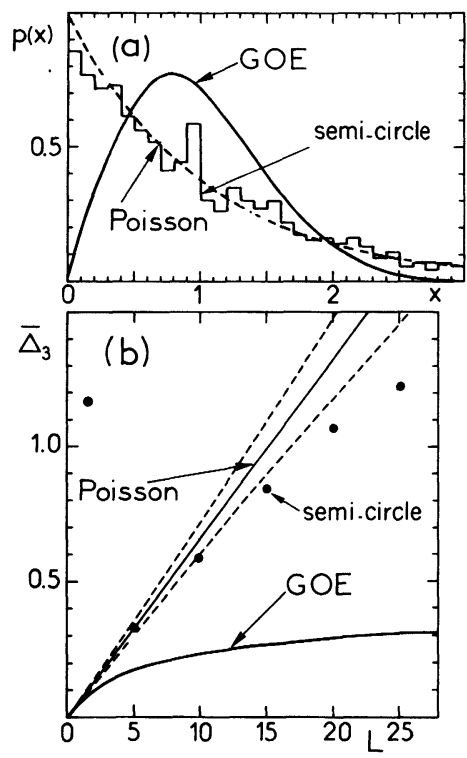

Fig. 5. - Same as in figure 2 for the eigenvalues of the semi-circular membrane. The stretch going from the 200th to the 1400 th eigenvalue has been used. Dashed lines for the $\bar{\Delta}_{3}$-value of Poisson indicate the effect of the finiteness of the sample. 
correlated spectra is to produce a Poisson spectrum. Consider, for instance, the effect of superposing at random $n$ picket fences (spectra of equally spaced levels). On the interval [0,1] one takes $n$ points $x_{i}(i=1,2, \ldots, n)$ at random uniformly distributed and one constructs an infinite spectrum by attaching to each point $x_{i}$ a picket fence of unit spacing. The resulting spectrum, in the limit of large $n$, is a Poisson spectrum. In the case of the semi-circular membrane we are in a similar situation. The eigenfrequencies $k_{n}\left(E_{n}=k_{n}^{2}\right)$ are given by the zeros of the Bessel functions $J_{v}(x)(v=1,2, \ldots)$. Let $j_{v, s}(s=1,2, \ldots)$ denote the $s$-th zero of $J_{v}(x)$. The $j_{v, s}$ s $(s=1,2, \ldots)$ extend from $\simeq v$ to infinity with a density

$$
\frac{1}{\pi} \sqrt{1-\frac{v^{2}}{k^{2}}} \quad \text { for } \quad k>v
$$

and are practically fluctuation-free. Consider now an interval at high frequency containing $N$ levels $k_{n}(n=1,2, \ldots, N)$ ordered with increasing value of $k_{n}$. The successive values of $k_{n}$ correspond to around $N$ different (and unordered) values of $v$. The point now is that zeros of $J_{v}$ and $J_{v^{\prime}}$ with $v$ not too close to $v^{\prime}$ are likely to be uncorrelated. Consider, for instance, a stretch of the eigenfrequencies near the 1400 th frequency. The labelling $(v, s)$ of successive eigenfrequencies is as follows : $(82,4),(70,7),(37,18),(45,15),(5,32),(32,20),(3,33),(1,34),(18,26),(60,10)$. We are therefore in a similar situation as when superposing picket fences, which leads to a Poisson spectrum.

In summary, the results presented here reinforce the conjecture that the spectrum of the Laplacian with Dirichlet (or Neumann) boundary conditions on an irregular boundary has asymptotically (high energy) GOE-fluctuations. By irregular we mean such that the corresponding classical billiard is a Bernoulli system (possibly that ergodicity is sufficient). To attack the conjecture theoretically (for attemps in this direction, see [13]) it may be convenient, instead of putting the complication in the shape of the boundary, to put it in the metric of the space and consider free motion without walls. We remind that the analogy between dispersing billiards and geodesic flows in spaces of negative curvature can be explained intuitively [14] and that the geodesic flow on a surface of negative curvature is a Bernoulli system [15]. At any rate, the following picture seems to emerge. At a " macroscopic scale ", we have universality properties of the spectrum of the Laplacian in a box : the average number of eigenvalues up to a given energy depends only on macroscopic features of the boundary, such as surface, perimeter. At the other extreme, at a " microscopic scale » (scale provided by the mean spacing), fluctuations also show universality patterns : Poisson-pattern for regular (integrable) systems, GOE-pattern for strongly chaotic systems. One expects that these results also apply in more than two dimensions, for instance to the electromagnetic oscillations of a cavity [16]. Measurements of a large number of eigenmodes of microwave cavities of irregular shapes, although difficult, seem to be feasible and are called for.

\section{References}

[1] Bohigas, O., Giannoni, M. J., Schmit, C., Phys. Rev. Lett. 52 (1984) 1.

[2] Bohigas, O., Giannoni, M. J., Schmit, C., in Proceedings of Conference on Quantum Chaos ed. by G. Casati, to appear;

Bohigas, O., Giannoni, M. J., in " Mathematical and Computational Methods in Nuclear Physics ", J. S. Dehesa, J. M. Gomez and A. Polls (eds.), Lecture Notes in Physics 209 (Springer-Verlag) 1984 ;

Schmit, C., in a Workshop on "Semiclassical Methods in Nuclear Physics ", J. Physique Colloq. 45 (1984) C6-379. 
[3] Porter, C. E., Statistical Theories of Spectra : Fluctuations (Academic Press) 1965 ;

Meнta, M. L., Random Matrices and the Statistical Theory of Energy Levels (Academic Press) 1967 ; Brody, T. A., Flores, J., French, J. B., Mello, P. A., Pandey, A., Wong, S. S. M., Rev. Mod. Phys. 53 (1981) 385.

[4] Berry, M. V., Tabor, M., Proc. R. Soc. London Ser. A 356 (1977) 375 ;

BERRY, M. V. in Chaotic Behaviour of Deterministic Systems, ed. by R. H. G. Helleman and G. Joos (North Holland) 1983.

[5] Haller, E., KöPPel, H., Cederbaum, L. S., Phys. Rev. Lett. 52 (1984) 1665 ;

Seligman, T. H., VerbaArschot, J. J. M., Zirnbauer, M. R., Phys. Rev. Lett. 53 (1984) 215.

[6] Bunimovich, L. A., Funct. Anal. Appl. 8 (1974) 254 ; Commun. Math. Phys. 65 (1979) 295.

[7] McDonald, S. W., Kaufman, A. N., Phys. Rev. Lett. 42 (1979) 1189.

[8] McDonald, S. W., Wave Dynamics of Regular and Chaotic Rays, Ph. D. Thesis, LBL-14837, 1983.

[9] Casati, G., Valz-Gris, F., Guarneri, I., Lett. Nuovo Cim. 28 (1980) 279.

[10] Baltes, H. P., Hilf, E. R., Spectra of Finite Systems (Bibliographisches Institut, Mannheim) 1976.

[11] RidDell Jr., R. J., Report LBL-5587 (1978);

LEPORE, J. V., RIDDELl Jr., R. J., Report LBL-3036 (1974).

[12] Bohigas, O., HaQ, R. U., Pandey, A., preprint.

[13] Pechukas, P., Phys. Rev. Lett. 51 (1983) 943 ;

BERRY, M. V. in Proceedings of "Conference on Quantum Chaos », ed. by G. Casati, to appear.

[14] ARnold, V. I., Russ. Math. Surv. 18 (1963) 85.

[15] ORnsteIn, D. S., Ergodic Theory, Randomness, and Dynamical Systems (Yale University Press) 1974.

[16] Dyson, F. J., private communication. 